\title{
INFLUENCE OF PULSE SHAPER GEOMETRY ON WAVE PULSES IN SHPB EXPERIMENTS
}

\author{
Robert Panowicz, Jacek Janiszewski, Krzysztof Kochanowski \\ Military University of Technology, Warsaw, Poland \\ e-mail: robert.panowicz@wat.edu.pl
}

\begin{abstract}
Results of numerical analysis of the influence of pulse shaper geometry on wave signals in the split Hopkinson pressure bar experiment are presented. Five pulse shapers, i.e. square, ring, cross, star and disk ones have been analysed. It has been assumed that the disc pulse shaper is the reference geometry to assess the remaining types of pulse shapers. The results of numerical analyses have shown that pulse shapers with shapes different than disk are highly capable of minimizing high-frequency Pochhammer-Chree oscillations and, thus, reduce dispersion of waves propagating in the bar. The greatest damping ability has been observed while using the ring pulse shaper at both low and high impact velocities of the striker.
\end{abstract}

Keywords: SHPB, pulse shaping technique, high-strain-rate testing, numerical analysis

\section{Introduction}

The Split Hopkinson Pressure Bar (SHPB) technique requires satisfying some fundamental conditions, i.e., one-dimensional wave propagation in bars, no friction between the specimen and the bars, negligibly low influence of inertia and nearly uniformly specimen deformation at a constant strain rate under dynamically equilibrated stresses (Chen and Song, 2011). The condition regarding the nearly uniformly specimen deformation under dynamically equilibrated stresses is satisfied when stresses on external contact surfaces of the specimen are equal.

The waveform of the phenomenon results in the fact that at the initial moment of loading the sample through the incident bar, the condition regarding the uniform deformation of the specimen under dynamically equilibrated stresses cannot be satisfied. This condition is satisfied after some time, depending on the shape of the incident pulse and on the pulse rising time as well as geometrical parameters of the specimen and its properties (Frantz and Follansbee, 1984; Follansbee, 1985; Gorham, 1991). Currently, the pulse shaper technique is widely used to shape the incident pulse. The technique is based on placing a small coaxial disk (pulse shaper) between the strike and the incident bar. This disk is made of a soft material compared to the striker and bars material, and deforms plastically when the striker hits it.

Frantz and Follansbee (1984) and Follansbee (1985) showed that with an increase in the shaper thickness, the pulse rise time increases and the amplitude of Pochhammer-Chree high-frequency oscillations (Pochhammer, 1876; Chree, 1886) decreases. As a result, the dispersion is reduced and the dynamic equilibrium in the specimen is achieved much faster.

In most cases, during experimental tests, different thickness and diameter of shapers made of copper were used to increase the rise time of the incident pulse to reduce dispersion as well as to achieve the state of dynamic equilibrium faster, see e.g. (Chen et al., 2003; Naghdabadi et al., 2012).

In SHPB, thin disks are used for wave shapers. This is due to, among others, the fact that it is easy to produce them with the use of machining methods or by punching them from metal sheets. For this reason, elements with more complex geometry, such as rings, crosses, stars, are 
not used. Hence, the idea of examining the influence of various shapes of pulse shapers on wave pulses registered in SHPB experiments.

The article presents results of numerical analyses for 5 types of geometry of the pulse shapers presented in Fig. 1.

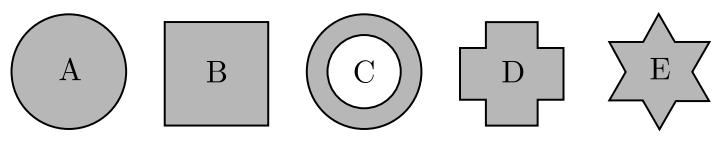

Fig. 1. Geometry of the analyzed pulse shapers

\section{Numerical modelling}

The model contains all main components of the arrangement: a pulse shaper, slide bearings and a barrel. In simulation, dimensions of all elements of SHPB are the same as in the experiments (Panowicz et al., 2017).

The Johnson-Cook constitutive model with the Gruneisen hydrodynamic equation of state has been used to describe the behavior of the copper shaper and a titanium specimen (Hallquist, 2006). The Ti6Al4V specimen ( $3.7 \mathrm{~mm}$ length, $4 \mathrm{~mm}$ diameter) and copper pulse shaper materials constants have been taken from literature (Grazka and Janiszewski, 2012; Ozel and Sima, 2010).

The authors used the Finite Element Method with a central difference time integration scheme implemented in explicit LS-Dyna to carry out numerical simulations (Hallquist, 2006).

In order to assess the correctness of the numerical model, validation with experimental tests for two striker impact velocities $(V=15.3 \mathrm{~m} / \mathrm{s}$ and $11.8 \mathrm{~m} / \mathrm{s})$ and two shaper thicknesses $(d=0.101$ and $0.201 \mathrm{~mm})$ has been carried out to obtain high compliance.

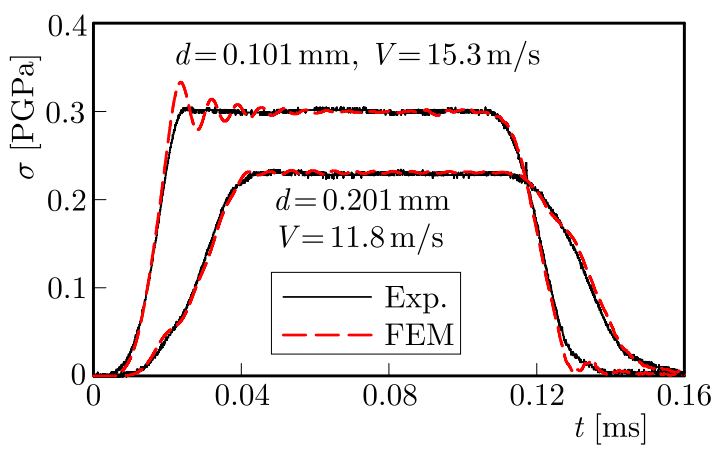

Fig. 2. Comparison of incident wave profiles obtained from numerical (FEM) and experimental (Exp.) analyses, in which shapers with different thicknesses d were used; the striker hits the bar with velocity $V$

\section{Results and discussion}

Numerical analysis of the geometry influence of the pulse shaper with thickness of $0.1 \mathrm{~mm}$ has been carried out for frequently used striker impact velocities, i.e. 10 and $15 \mathrm{~m} / \mathrm{s}$. The results are presented in the form of incident and reflect waves pulses shown in Figs. 3 and 4, respectively.

Based on the obtained results, the influence of the pulse shapers geometry on waves pulses profiles has been confirmed, however, it is little, especially in the test with velocity of $10 \mathrm{~m} / \mathrm{s}$ (Figs. 3a,b and 4a,b). This effect is also manifested in the course of both the rising and falling time of wave profiles. In relation to the standard geometry of the pulse shaper - the disk (geometry A, Fig. 1), the profiles of the rising and falling time of the incident wave, for the remaining types of pulse shapers, have a characteristic inflection occurring at about $2 / 3$ of the wave amplitude. The rise time of the wave profile to the point of inflection is shorter in relation 
(a)

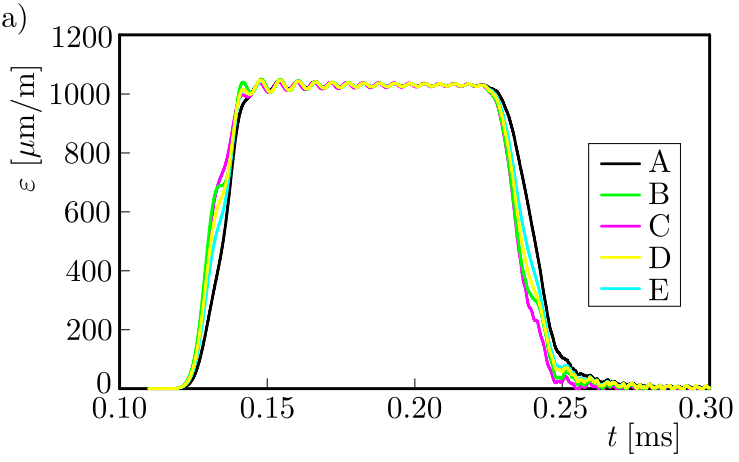

(c)

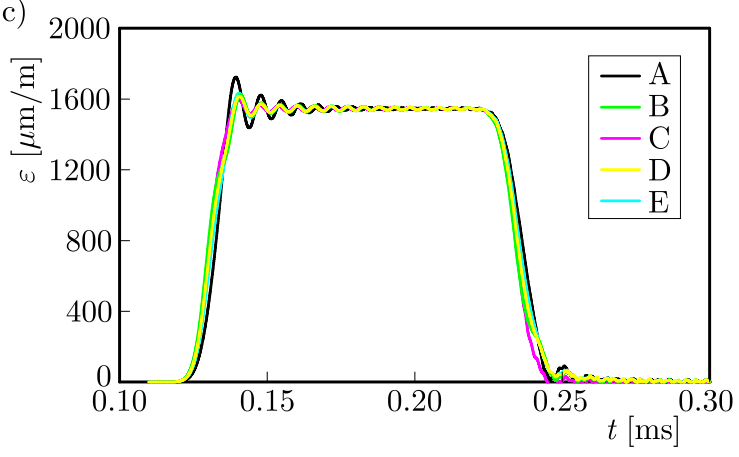

(b)

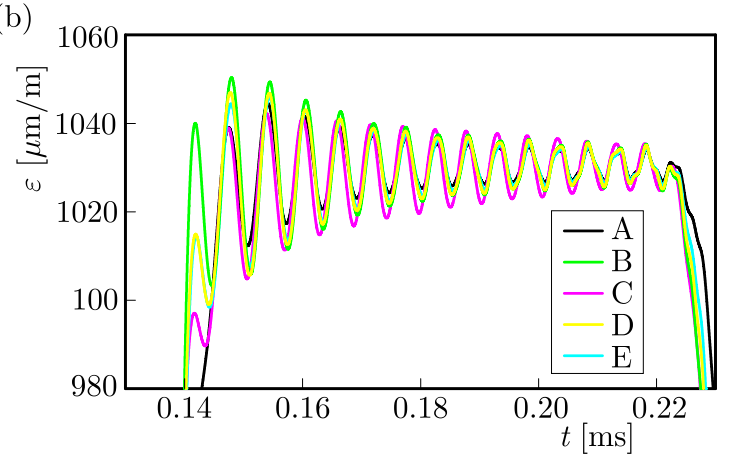

(d)

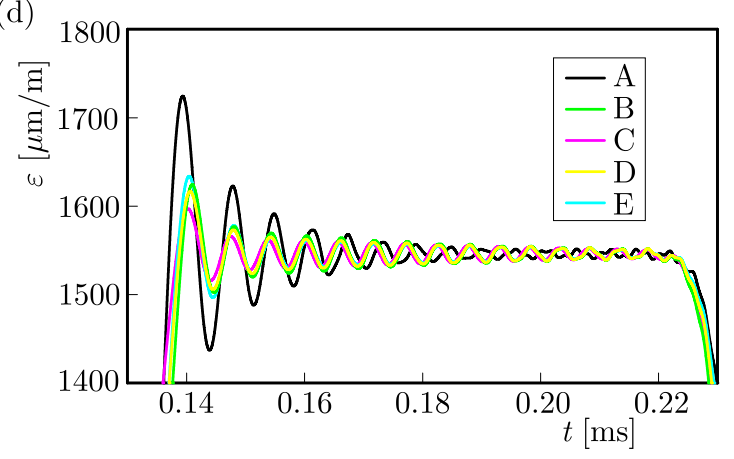

Fig. 3. Incident pulses for different striker velocity: (a), (b) $V=10 \mathrm{~m} / \mathrm{s}$, (c), (d) $V=15 \mathrm{~m} / \mathrm{s}$, (a) and (c) all signals, (b) and (d) zoom

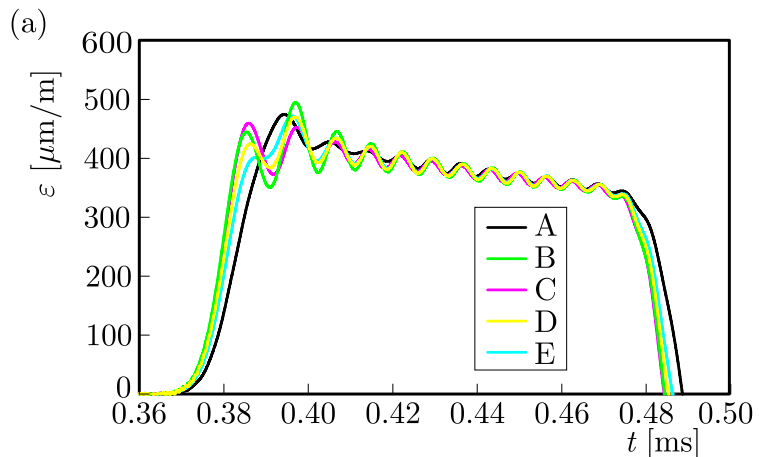

(c)

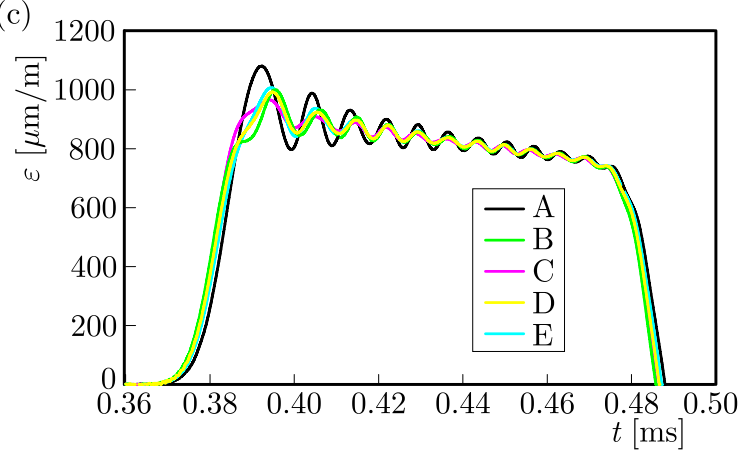

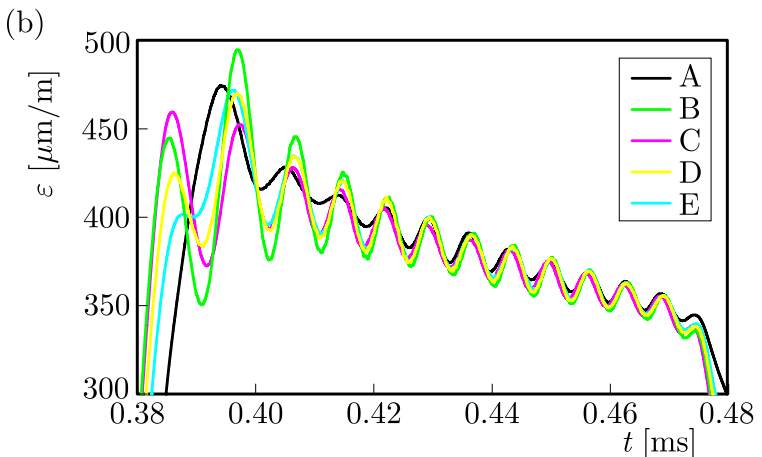

(d)

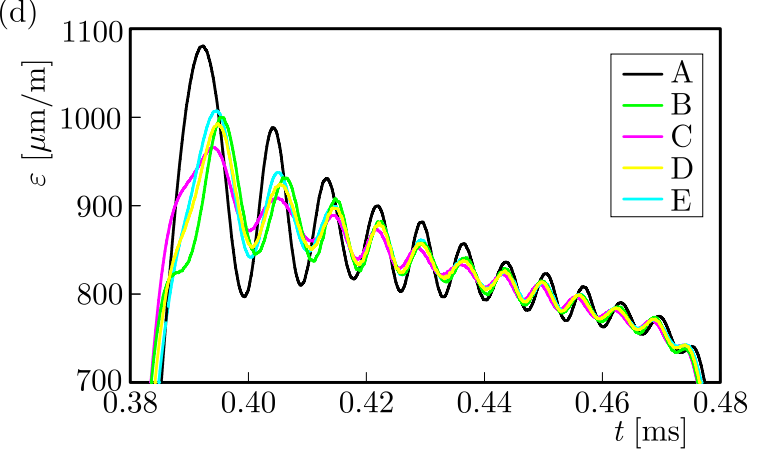

Fig. 4. Reflected pulses for different striker velocity: (a), (b) $V=10 \mathrm{~m} / \mathrm{s}$, (c), (d) $V=15 \mathrm{~m} / \mathrm{s}$, (a) and (c) all signals, (b) and (d) zoom

to the rise time when the classical shaper is used (geometry A, Fig. 1). This is due to a faster process of deformation and work hardening of the pulse shapers.

Small differences can also be observed in the area of the occurrence of Pochhammer-Chree high-frequency oscillations. The highest oscillation amplitude for the striker velocity of $10 \mathrm{~m} / \mathrm{s}$ 
was found for the square geometry, and the smallest for the ring geometry. In turn, for the striker impact velocity equal to $15 \mathrm{~m} / \mathrm{s}$, the largest oscillation amplitude occurs in the case when the pulse shaper of the disk type is used, and the smallest for the ring type. Therefore, it can be concluded that the pulse shaper with ring (Fig. 1c) geometry has the highest ability to suppress high-frequency components of the pulses and, thus, to minimize dispersion of waves propagating in the SHPB bar.

It should be noted that the effect of the pulse shaper geometry is revealed with an increase of the striker impact velocity. At the striker velocity equal to $15 \mathrm{~m} / \mathrm{s}$, the high damping ability is demonstrated by pulse shapers with geometry different than the disk (Fig. 1a). It follows that, especially for high striker velocities, the use of a classical pulse shaper with disk geometry is not appropriate. It is also observed in the reflected wave profile (Fig. 4c,d) on which the largest oscillations amplitudes are found for the disk pulse shaper (geometry A). Too large oscillation amplitudes visible on the wave profile delay the attainment of the specimen dynamic stress equilibrium state and, thus, make it impossible to meet the basic methodological requirement of the SHPB technique.

\section{Conclusion}

A method frequently used in the SHPB technique for forming an incident wave propagating in the input bar is the use of a pulse shaper with disk geometry. The numerical analysis presented in the article shows, however, that it is advantageous to use pulse shapers with shapes different than disks. It has been found that they have a high ability to minimize high-frequency Pochhammer-Chree oscillations and, thus, reduce dispersion of waves propagating in the bar. For example, a ring pulse shaper has a greater ability to suppress oscillations than a disk pulse shaper at both low and high striker impact velocities. The ring pulse shaper also allows reaching the equilibrium stress state in the test specimen faster. This feature is really desirable, especially while testing high-strength materials with low plastic properties.

\section{Acknowledgements}

The support of Military University of Technology grants PBS 23-937 and 23-941 is gratefully acknowledged.

\section{References}

1. Chen W., Song B., 2011, Split Hopkinson (Kolsky) Bar: Design, Testing and Applications, Berlin, Springer

2. Chen W., Song B., Frew D.J., Forrestal M.J., 2003, Dynamic small strain measurements of a metal specimen with a split Hopkinson pressure bar, Experimental Mechanics, 43, 1

3. Chree C., 1886, Longitudinal vibrations of a circular bar, Quarterly Journal of Pure and Applied Mathematics, 21, 287-298

4. Follansbee P.S., 1985, The Hopkinson Bar in Mechanical Testing and Evaluations, ASM Handbook, 9th ed. ASM Int., Materials Park Ohio

5. Frantz C.E., Follansbee P.S., 1984, Experimental techniques with the split Hopkinson pressure bar, Proceedings of the 8th International Conference on High Energy Rate Fabrication, San Antonio, Texas, TX, 229-236

6. Frew D.J., Forrestal M.J., Chen W., 2002., Pulse shaping techniques for testing brittle materials with a split Hopkinson pressure bar, Experimental Mechanics, 42, 93-106 
7. Gorham D.A., 1991, The effect of specimen dimensions on high strain rate compression measurements of copper, Journal of Physics, D: Applied Physics, 24, 8, 1489-1492, https://doi.org/ $10.1088 / 0022-3727 / 24 / 8 / 041$

8. Grazka M., Janiszewski J., 2012, Identification of Johnson-Cook equation constants using finite element method, Engineering Transactions, 60, 215-223

9. Hallquist J.O., 2006, Ls-Dyna. Theoretical Manual, California: Livermore Software Technology Corporation

10. Naghdabadi R., Ashrafi M.J., Arghavanic J., 2012, Experimental and numerical investigation of pulse-shaped split Hopkinson pressure bar test, Materials Science and Engineering A, 539, $285-293$

11. Nemat-Nasser S., IsaAcs J.B., Starrett J.E., 1991, Hopkinson techniques for dynamic recovery experiments, Proceedings of the Royal Society, 435, 371-391

12. Ozel T., Sima M., 2010, Finite element simulation of high speed machining Ti-6Al-4V alloy using modified material models, Transactions of NAMRI/SME, 38, 49-56

13. Panowicz R., Janiszewski J., Traczyk M., 2017, Strain measuring accuracy with splitting-beam laser extensometer technique at split Hopkinson compression bar experiment, Bulletin of the Polish Academy of Sciences Technical Sciences, 65, 2, 163-169, https://doi.org/10.1515/bpasts2017-0020

14. Pochhammer L., 1876, On the propagation velocities of small oscillations in an unlimited isotropic circular cylinder, Journal für die reine und angewandte Mathematik, 81, 324-326 